\title{
The Distance Between Us: the COVID-19 Pandemic's Effects on Burnout Among Resident Physicians
}

\author{
Kelsey H. Natsuhara ${ }^{1}$ (D) Hala T. Borno ${ }^{2}$ (D) \\ Accepted: 27 September 2021 / Published online: 15 October 2021 \\ (c) International Association of Medical Science Educators 2021
}

\begin{abstract}
Entering the second year of the COVID-19 pandemic, we reflect on how this public health crisis has amplified burnout in the medical profession. In particular, the pandemic has had a significant impact on medical residents. Recognizing trainee burnout as a side effect of the pandemic is crucial and highlights the need for programmatic change to support medical trainees. We reviewed the literature and propose multiple interventions to improve trainee well-being, targeting individual, peer-to-peer, and system levels. The pandemic has highlighted the importance of institutional support for medical trainees to prevent burnout and protect the pipeline of future physicians.
\end{abstract}

Keywords Burnout · Medical education · COVID-19 · Well-being

\section{Introduction}

Over a year has passed since the word "coronavirus" entered the global lexicon. The past months have not only been marked by the personal challenges of the COVID-19 pandemic, but also the unforeseeable professional ones. As physicians, we have become accustomed to the rhythm of donning and doffing personal protective equipment. We have called too many families to tell them their loved ones were dying, with only a video call to offer to say goodbye. Some of us have contracted the virus. We have cried and grappled with the lack of control and immense loss this pandemic has unleashed. All of this has taken its toll.

During this pandemic, physicians have often been portrayed as martyrs; however, less thought is given to our feelings of vulnerability. We have experienced burnout in medical training before, as have most of our peers at one point or another. However, this year is different and places immense hardship on physicians. In particular, physicians

Kelsey H. Natsuhara

kelsey.natsuhara@ucsf.edu

1 Department of Medicine, Division of General Internal Medicine, University of California, San Francisco, CA, USA

2 Department of Medicine, Division of Hematology/Oncology, University of California, San Francisco, CA, USA in training, who face high clinical burden, are at substantial risk for burnout in this pandemic. Early studies have begun to characterize the impact of COVID on medical residents, ranging from concerns on the impact on their education and decreased exposure to non-COVID diseases [1], fear of contracting COVID or spreading the virus to their families [2], and reports of increased rates of burnout among residents caring for COVID-19 patients [3].

While the pandemic has undoubtedly affected medical trainees of all levels (i.e., medical students, residents, and fellows), this manuscript is principally focused on the medical resident experience. In this commentary, we review the existing literature on physician burnout and highlight the unique challenges and stressors that are placed on resident physicians during the current pandemic.

\section{Physician Burnout During the Pandemic}

Burnout is defined as a low sense of personal accomplishment, emotional exhaustion, cynicism, and depersonalization [4]. Burnout is widespread in the medical field with physicians experiencing burnout at a higher rate than the general population [4]. As shown in Table 1, the pandemic has amplified all aspects in the definition of burnout, but the depersonalization is most profound. During this pandemic, we watch case statistics broadcast on the news. However, the numbers cannot do justice to the individual stories of suffering. The 
Table 1 The effect of burnout on resident physicians during the COVID-19 pandemic

\begin{tabular}{|c|c|c|}
\hline Components of burnout ${ }^{*}$ & Conditions in training that amplify burnout & COVID's effects on burnout \\
\hline $\begin{array}{l}\text { Low sense of personal } \\
\text { accomplishment }\end{array}$ & $\begin{array}{l}\text { - Limited recognition or reward for hard work } \\
\text { - Several high stakes responsibilities with insufficient } \\
\text { ancillary support or acknowledgement }\end{array}$ & $\begin{array}{l}\text { - Challenge of clinical work with treating novel SARS-CoV-2 } \\
\text { and moral distress from making decisions with limited } \\
\text { knowledge about the disease } \\
\text { - Rising case numbers despite healthcare workers' } \\
\text { attempts at public education }\end{array}$ \\
\hline Emotional exhaustion & $\begin{array}{l}\text { - Heavy workload and high duty hours } \\
\text { - Lack of time for personal and family support } \\
\text { - Limited time off with little time to reflect and recover } \\
\text { - Witnessing pain, suffering, and death }\end{array}$ & $\begin{array}{l}\text { - High rates of death and dying leading to the frequent } \\
\text { end of life conversations and the need to support } \\
\text { patients and families, often via telephone } \\
\text { - Lack of childcare during the pandemic leading to } \\
\text { increased responsibilities at home }\end{array}$ \\
\hline Cynicism & $\begin{array}{l}\text { - Lack of control over schedules and roles } \\
\text { - Assumption that residency is supposed to be this hard }\end{array}$ & $\begin{array}{l}\text { - Repeated "surges" in cases } \\
\text { - Lack of societal acceptance of mask-wearing and social } \\
\text { distancing leading to a higher number of cases }\end{array}$ \\
\hline Depersonalization & $\begin{array}{l}\text { - Frequently changing clinical roles on different rotations } \\
\text { - Needing to distance from patients to emotionally protect } \\
\text { oneself from repeated grief and trauma of death and } \\
\text { dying }\end{array}$ & $\begin{array}{l}\text { - Social distancing and mask-wearing lead to challenges } \\
\text { connecting with patients and colleagues } \\
\text { - Inability of patients to have visitors in the hospital can } \\
\text { dehumanize patients and families } \\
\text { - End of life conversations and family visits via phone } \\
\text { and video call feel inadequate }\end{array}$ \\
\hline
\end{tabular}

*According to Maslach definition of burnout [1]

imperative of social distance to keep us safe has meant that distance has inevitably grown in our relationships, too. The act of wearing a mask depersonalizes us, hides our identity, and often our emotions. We feel this loss of identity most in the hospital. We miss smiling at our patients, sitting with their families, and sharing in their grief and hope. Telemedicine has its role to facilitate communication, but it cannot replace holding the hand of a loved one or hearing a last breath.

We recognize that this depersonalization especially affects our newest generation of physicians and learners-who know only how to be a doctor during a pandemic. They are taught to distance: from their patients and their colleagues in order to stay safe. Workrooms have been reconfigured, so we sit further apart. Teaching conferences and social gatherings are held online- - too easy to mute, turn off video, and disengage. For trainees during this era, burnout is amplified due to this lack of meaningful human interaction. We must recognize trainee burnout as yet another consequence of COVID and be thoughtful about how to support this generation of physicians and learners.

\section{Physician Mental Health}

Even before the pandemic, burnout and mental health were critical problems facing clinicians. In a study of over 7000 US physicians, 38\% screened positive for depression [4]. More concerning is that burnout and depression particularly affect younger physicians, with rates of burnout among residents and fellows estimated at 60\% [5]. Burnout has also been associated with increased suicide rates, and each year, it is estimated that 400 US physicians die by suicide [6].
Furthermore, previous public health disasters like the 2003 SARS outbreak and 2014 Ebola outbreak have demonstrated that epidemic events are associated with increased healthcare worker distress and psychiatric morbidity [7, 8]. The COVID19 pandemic is no exception. In a recent survey of over 1000 intensivists caring for COVID patients, rates of burnout were reported at 51\% [9]. Some have suggested that the challenges of making clinical decisions with limited information contribute to moral distress and burnout [9]. Consistent with this, early studies have shown that the psychological burden is higher in younger, less experienced healthcare providers, highlighting the need to support residents in training [10]. In a recently published international study of over 1400 residents and fellows, trainees who cared for a greater number of patients with COVID-19 were more likely to report burnout [3].

Even before the pandemic, it was challenging in medical training to provide structural supports, in terms of protected time and skill-building, to consistently cultivate well-being. Now, amidst a pandemic, helping residents develop skills to avoid burnout can feel like a daunting task for residency programs. However, given data that younger physicians are at higher risk for burnout, a focus on teaching medical trainees the skills to cope with burnout has never been more critical.

\section{Strategies to Improve Trainee Well-being}

Interventions to target resident well-being are often focused on individual skill-building, such as mindfulness or stress management $[11,12]$. However, in an environment where trainees have limited time, placing the burden 
on individuals to seek out these resources is likely inadequate. Institutional support and programmatic change are necessary to prevent burnout. We propose a multi-level approach that targets interventions at the individual, peerto-peer, and system levels to support trainee well-being in this pandemic (Fig. 1).

Given the depersonalization inherent in this pandemic, we must be thoughtful about how to facilitate social support for trainees. In the literature, it has been described that interns rely heavily on their senior residents and near-peers for guidance and support [13]. Moore et al. recently described a longitudinal peer support curriculum during residency that focuses on teaching senior residents to build peer-support skills, rather than developing these communication frameworks by trial and error [14]. A model like this can equip senior residents with the skills they need to support more junior residents. Additionally, because we know that emotional support often happens at the peer-to-peer level, programs should ensure that there is a safe mechanism where trainees can relay their concerns about their peers who may be struggling or need more support [13]. Creating a channel to identify high-risk individuals may allow programs to better target their interventions. Moreover, this type of curriculum can be easily adapted to a virtual platform by providing skillbuilding curriculum over video conference and facilitating one-on-one peer support by connecting residents via video or phone calls, text messages, or arranging one-on-one COVID safe meetings outdoors.

Facilitated small group curricula have also been shown to reduce burnout domain scores [15]. Programs should operationalize consistent sessions on burnout that highlight the unique challenges residents face. This process, even over video, normalizes burnout and creates space for connection. Providing protected time for interns to debrief, freed from their pagers and other clinical duties, demonstrated programs' commitment and prioritization of trainee well-being.

Though helping trainees make time for reflection is pivotal, it is not enough. Training programs should seek to make mental health resources accessible and, ideally, free of cost to their trainees. We need to invest in mental health resources, and this includes more time off from clinical duty. The well-being of our physician pipeline must be prioritized to ensure sustainability and reduce attrition from the clinical workforce.

Furthermore, we must address the new challenges of caring for patients both in the hospital and in clinic using telehealth. Both settings present unique challenges for telecommunication. In the outpatient setting, trainees must communicate with patients over virtual platforms, as telehealth has grown into a larger part of our careers as physicians. In the hospital, trainees must navigate caring for patients with visitor restrictions due to infection control precautions and often conduct complex goals of care meetings via phone or video. While most physicians report positive experiences from caring for patients at the end of life, the emotional impact of witnessing death weighs heavy and is amplified in this pandemic [16]. It is critical to implement specific training for physicians in telecommunication to ease the burden of these conversations by arming trainees with the skills and resources necessary to care for patients. Telecommunication

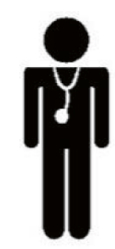

- Proactive assessment of trainee well-being

Individual - Improving access to mental health resources

level - Clear and well communicated family leave policies

- Subsidies for childcare or provision of childcare resources

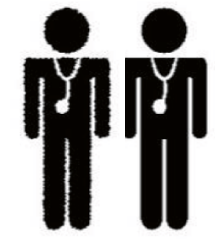

Peer to - Facilitated small group curricula

Peer - Near peer longitudinal support curriculum

- Safe mechanism to relay concerns about their peers' well-being

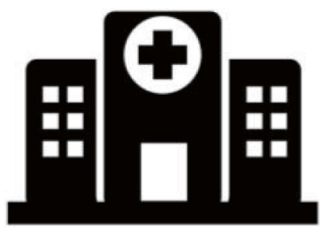

- Telecommunication training

Systems- - Investment in physical resources (i.e. tablets, speakers)

Wide - Anti-racism training

- Continuous assessment of institutional culture

Fig. 1 Multi-level interventions to reduce burnout among resident physicians during the COVID-19 pandemic 
training for physicians should teach residents to communicate with both patients and their families over virtual platforms, with a specific focus on conducting goals of care conversations virtually. In addition, investing in hardware, like webcams, tablets, and speakers is essential to equip residents with the tools they need to care for patients. Telemedicine will undoubtedly remain a substantial part of our careers as physicians and investing in resources and training to facilitate this new form of communication is key.

Additionally, programs should institute mechanisms to proactively assess the institutional culture and well-being among their trainees to identify issues that are contributing to burnout. For example, frequent surveys, focus groups, or forming a resident council to monitor the challenges experienced among trainees, may allow programs to target issues leading to burnout and identify individuals most at risk [17]. These types of interventions are relevant in non-pandemic times; however, the current pandemic highlights the critical need for a system of ongoing assessment that will allow programs to appropriately leverage resources. Furthermore, times of disruption to our typical systems and workflows, often serve as an opportunity for innovation. This pandemic may present an opportunity for training programs to assess residents' needs and make changes to support trainee wellness when healthcare systems and the structure of training programs may be more malleable and amenable to change.

\section{Disparities in the Workforce}

In addition, disparities in the workforce should be addressed to ensure that institutions do not inadvertently perpetuate these issues. Women physicians are more likely to face genderrelated barriers to professional advancement and bear more responsibility for household tasks and childcare, all of which contribute to higher rates of burnout compared to men [18]. In particular, the pandemic has had huge effects on childcare, which disproportionately affects female trainees [19]. Thus, providing resources for family support is critical. Chesak and colleagues emphasize the importance of well-communicated family leave policies, provision of childcare resources, or subsidies for childcare costs as potential areas for intervention to reduce burnout among women physicians [20]. Programs could consider offering grants or loans to trainees who have been disproportionately affected by the pandemic, specifically targeting trainees whose childcare expenses increased due to daycare closures and needing to hire additional help at home [20, 21]. Additionally, programs could consider building upon leave policies, like the federal Families First Coronavirus Response Act, that extends additional paid time off for parents who need to provide childcare due to school or childcare closures related to COVID-19 [22, 23].
Secondly, it is important to recognize that only $4 \%$ of physicians come from underrepresented minorities and are more likely to serve vulnerable patient populations [18]. This is especially important given the pandemic has disproportionately impacted Black and Hispanic communities. Furthermore, this past year has highlighted the structural racism deeply embedded within our society. The possibility of burnout and physician attrition from underrepresented minorities is likely to affect these communities most. Thus, it is also important to focus on anti-racism education and training. In the literature, structural competency frameworks that focus on helping physicians recognize "health and illness as the downstream effects of broad social, political, and economic structures" and equip them to effectively respond have shown promise [24]. Gray et al. recommend a multi-pronged approach including education, funding for community-based research on structural racism, and forming community councils to review institutional health equity initiatives and provide feedback [25].

\section{Conclusion}

The COVID-19 pandemic has amplified all aspects of burnout and had a significant effect on medical trainees. The above interventions targeting well-being and burnout at the individual, peer-to-peer, and system-wide levels can serve as a framework for programs to improve trainee well-being in this unprecedented time. We must call attention to the depersonalization and heightened burnout that our medical residents are facing.

The interventions we propose cover multiple domains from improving access to mental health resources, expanding childcare support for trainees, developing peer support curriculum, and improving telecommunication tools and training. We believe that, while these interventions are especially important during pandemic years, they will also be relevant in the future to promote trainee wellness. Physician burnout was widespread in the medical field prior to the pandemic and will likely continue to be an issue in the future. The pandemic is a time of disruption in our daily lives and systems and also serves as an opportunity to rethink our structures of training and better support medical residents.

In the news, clinicians have been called superheroes. However, this may be counterproductive. While the sentiment is appreciated, it perpetuates the notion that physicians are supposed to be strong and unbreakable, when in reality, this year has been profoundly challenging. What resident physicians need most are concrete support and resources. Throughout a pandemic that has changed what it means to be a doctor, we must embrace our collective responsibility to support the next generation of physicians. 
Author Contribution All authors contributed to the manuscript conception. The first draft of the manuscript was written by $\mathrm{KN}$, and all authors commented on previous versions of the manuscript. All authors read and approved the final manuscript.

Availability of Data and Material Data sharing is not applicable to this article as no datasets were generated or analyzed during the current study.

\section{Declarations}

Ethics Approvals This is a commentary piece without participant data. As such, no ethical approval was required.

Consent to Participate/Publish No participant data was used in this study. No informed consent was obtained.

Conflict of Interest The authors declare no competing interests.

\section{References}

1. Khalafallah AM, Lam S, Gami A, et al. A national survey on the impact of the COVID-19 pandemic upon burnout and career satisfaction among neurosurgery residents. J Clin Neurosci. 2020;80:137-42.

2. Aziz H, James T, Remulla D, et al. Effect of COVID-19 on surgical training across the United States: a national survey of general surgery residents. J Surg Educ. 2021;78(2):431-39.

3. Cravero AL, Kim NJ, Feld LD, et al. Impact of exposure to patients with COVID-19 on residents and fellows: an international survey of 1420 trainees. Postgrad Med J. 2020.

4. Shanafelt TD, Boone S, Tan L, et al. Burnout and satisfaction with work-life balance among US physicians relative to the general US population. Arch Intern Med. 2012;172(18):1377-85.

5. Dyrbye LN, West CP, Satele D, et al. Burnout among U.S. medical students, residents, and early career physicians relative to the general U.S. population. Acad Med. 2014;89(3):443-51.

6. Stehman CR, Testo Z, Gershaw RS, et al. Burnout, drop out, suicide: physician loss in emergency medicine, part I. West J Emerg Med. 2019;20(3):485-94.

7. Chong MY, Wang WC, Hsieh WC, et al. Psychological impact of severe acute respiratory syndrome on health workers in a tertiary hospital. Br J Psychiatry. 2004;185:127-33.

8. Raven J, Wurie H, Witter S. Health workers' experiences of coping with the Ebola epidemic in Sierra Leone's health system: a qualitative study. BMC Health Serv Res. 2018;18(1):251.

9. Azoulay E, De Waele J, Ferrer R, et al. Symptoms of burnout in intensive care unit specialists facing the COVID-19 outbreak. Ann Intensive Care. 2020;10(1):110.
10. Elbay RY, Kurtulmuş A, Arpacioğlu S, et al. Depression, anxiety, stress levels of physicians and associated factors in COVID-19 pandemics. Psychiatry Res. 2020;290:113130.

11. Milstein JM, Raingruber BJ, Bennett SH, et al. Burnout assessment in house officers: Evaluation of an intervention to reduce stress. Med Teach. 2009;31(4):338-41.

12. Goldhagen BE, Kingsolver K, Stinnett SS, et al. Stress and burnout in residents: impact of mindfulness-based resilience training. Adv Med Educ Pract. 2015;6:525-32.

13. Baldwin DC, Jr., Daugherty SR. How residents say they learn: a national, multi-specialty survey of first- and second-year residents. J Grad Med Educ. 2016;8(4):631-39.

14. Moore KA, O'Brien BC, Thomas LR. I wish they had asked: a qualitative study of emotional distress and peer support during internship. J Gen Intern Med. 2020;35(12):3443-48.

15. West CP, Dyrbye LN, Erwin PJ, et al. Interventions to prevent and reduce physician burnout: a systematic review and meta-analysis. Lancet. 2016;388(10057):2272-81.

16. Redinbaugh EM, Sullivan AM, Block SD, et al. Doctors' emotional reactions to recent death of a patient: cross sectional study of hospital doctors. BMJ. 2003;327(7408):185.

17. Montgomery A, Todorova I, Baban A, et al. Improving quality and safety in the hospital: the link between organizational culture, burnout, and quality of care. Br J Health Psychol. 2013;18(3):656--62.

18. Silver JK, Bean AC, Slocum C, et al. Physician workforce disparities and patient care: a narrative review. Health Equity. 2019;3(1):360-77.

19. Kannampallil TG, Goss CW, Evanoff BA, et al. Exposure to COVID-19 patients increases physician trainee stress and burnout. PLoS One. 2020;15(8):e0237301.

20. Chesak SS, Cutshall S, Anderson A, et al. Burnout among women physicians: a call to action. Curr Cardiol Rep. 2020;22(7):45.

21. Joshi A, Garver KA, Balasubramanian S, et al. Childcare for radiology workers during the COVID-19 pandemic: no small matter. J Am Coll Radiol. 2020;17(11):1532-34.

22. Pichler S, Wen K, Ziebarth NR. COVID-19 emergency sick leave has helped flatten the curve in the United States: study examines the impact of emergency sick leave on the spread of COVID-19. Health Affairs. 2020;39(12):2197-204.

23. Vazquez J, Islam T, Beller J, et al. Expanding paid sick leave as a public health tool in the COVID-19 pandemic. J Occup Environ Med. 2020;62(10):e598-9.

24. Neff J, Holmes SM, Knight KR, et al. Structural competency: curriculum for medical students, residents, and interprofessional teams on the structural factors that produce health disparities. MedEdPORTAL. 2020;16:10888.

25. Gray DM, Joseph JJ, Glover AR, et al. How academia should respond to racism. Nat Rev Gastroenterol Hepatol. 2020;17(10):589-90.

Publisher's Note Springer Nature remains neutral with regard to jurisdictional claims in published maps and institutional affiliations. 